\title{
Positively disruptive
}

\section{Companies now provide individuals with their own genotypes and associated risks extrapolated from association studies. The best outcomes would be to convert patients into active investigators and navigators of their own health, to make genetics the foundation of medical education and to expand the scope of genetic counseling as a profession.}

W e (Editorial, December 2007) and a recent Perspective (New Engl. J. Med. 358, 105-107; 2008) have cautioned users of new consumer genomics services such as deCODEme and Navigenics that variants with low relative risk-including most of the SNPs associated with common diseases_-make poor classifiers. So, individual genomics may not induce anyone to take a clinical test until the growing list of risk variants adds up to the point where it can identify some particularly unlucky individuals. However, judging by the Perspective, the services have caught physicians right in the middle of their efforts to bring genetics to the core of medical education (Ann. Rev. Genomics Hum. Genet. 6, 313-330; 2005), causing them to worry that they will soon be deluged with patients asking them what all this 'premature' risk prediction means for them.

It is not beyond most physicians' skills to explain the quantitative risks conferred by - and the research underlying — the health predictors they currently use: BMI, cholesterol, blood pressure, age and sex. A physician can also relate quantitative risks conferred by a family history containing one or more affected relatives and should also be able to advise the patient whether or not to participate in research tests, such as that for C-reactive protein, with scientific reasons. It is to be expected that an individual's risk profile will change with each new study, as new variants will be discovered that moderate or exacerbate their individual risk, but this pace of new discoveries should not be daunting to continuing medical education. Blood pressure and BMI limits now elicit more vigorous treatment from physicians, as better information has been gained.

Generally effective environmental interventions are currently recommended to patients and 'healthcare consumers' without reference to genotypic information. However, to give one example, different people become hypertensive by different routes of genetic and environmental exposures, and it may take years of personal experimentation with different drugs, doses and dose regimens to achieve a balance of blood pressure control and side effects. Optimizing generally applicable interventions with genomic information and with participation of the research subjects themselves may be the best way forward. Intellectually motivated people may even be spurred to exercise out of curiosity, in order to see whether they can fulfill the promise of their 'elite athlete' SNPs.

Giving individuals their own genotype is not so much premature as truly disruptive. The individual gains a personal stake in the ongoing research effort and a huge incentive to find out more. A personal stake in finding out something that was not previously known is the key to getting students into research and may well be a powerful tool to educate and interest members of the public in the details of their own health and functioning. This boon was anticipated two years ago by George Church (Mol. Syst. Biol. 1:2005.0030; doi:10.1038/msb4100040), at the launch of the 'collaborative research endeavor' called the Personal Genome Projects (http://www.personalgenomes.org/). For many, the personal genomic profile is the stimulus to explore family history. Discussion of personal genetic risk factors may act to release family medical history kept private because it had no perceived use.

The pressure of information also creates a need for genetic counselors, but if uptake and use of individual genomics spreads as fast or widely as it seems likely to do, the counseling curriculum will undergo a rapid shift of emphasis away from rare mendelian diseases to both rare and common genetic determinants of common diseases and will acquire a new set of courses to deal with evaluating environmental risks.

Sometimes, the best doctor will say "we don't know yet, let's find out together." It is right to be skeptical of unknowns, but it would be wrong to underestimate the motivational potential inherent in handing people their genomes and asking them to participate in finding out more about their variation and phenotypes. With no personal stake and no current health problems, it may not occur to many individuals that their participation in longitudinal research would be welcomed by scientists and the public alike.

In the meantime, individual genomics will have informed thousands participating in one of the most exciting areas of biomedical research, and it may recruit participants in prospective studies that they will have funded partially from their own pockets. That being said, they are co-investigators, not patients, and the experiment will be conducted on their own terms! 